\title{
Determining the Best Drought Tolerance Indices using Artificial Neural Network (ANN): Insight into Application of Intelligent Agriculture in Agronomy and Plant breeding
}

\author{
A. Etminan ${ }^{1 *}$, A. Pour-Aboughadareh ${ }^{2}$, R. Mohammadi ${ }^{3}$, L. Shooshtari ${ }^{1}$, \\ M. YOUSEFIAZARKHANIAN ${ }^{4}$ and H. MORADKHANI ${ }^{1}$ \\ ${ }^{1}$ Department of Biotechnology and Plant Breeding, Kermanshah Branch, Islamic Azad University, \\ Kermanshah, Iran \\ ${ }^{2}$ Department of Agronomy and Plant Breeding, Faculty of Agriculture, University of Tehran, Karaj, Iran \\ ${ }^{3}$ Dryland Agricultural Research Institute, Sararood branch, Agricultural Research, \\ Education and Extension Organization (AREEO), Kermanshah, Iran \\ ${ }^{4}$ Agricultural Jihad Organization, Qazvin, Iran
}

(Received 27 November 2017; Accepted 25 June 2018; Communicated by S. Gottwald)

\begin{abstract}
In the present study, efficiency of the artificial neural network (ANN) method to identify the best drought tolerance indices was investigated. For this purpose, 25 durum genotypes were evaluated under rainfed and supplemental irrigation environments during two consecutive cropping seasons (2011-2013). The results of combined analysis of variance (ANOVA) revealed that year, environment, genotype and their interaction effects were significant for grain yield. Mean grain yield of the genotypes ranged from $184.93 \mathrm{~g} \mathrm{plot}^{-1}$ under rainfed environment to $659.32 \mathrm{~g} \mathrm{plot}^{-1}$ under irrigated environment. Based on the ANN results, yield stability index (YSI), harmonic mean (HM) and stress susceptible index (SSI) were identified as the best indices to predict drought-tolerant genotypes. However, mean productivity (MP) followed by geometric mean productivity (GMP) and HM were found to be accurate indices for screening drought tolerant genotypes. In general, our results indicated that genotypes G9, G12, G21, G23 and G24 were identified as more desirable genotypes for cultivation in drought-prone environments. Importantly, these results could provide an evidence that ANN method can play an important role in the selection of drought tolerant genotypes and also could be useful in other biological contexts.
\end{abstract}

Keywords: drought stress, selection indices, ANN analysis, durum wheat

\section{Introduction}

Durum wheat (Triticum turgidum L. var. durum Desf.) is an important cereal crop, which accounts for approximately $10 \%$ of the total wheat production, inhabiting around 20 million hectares worldwide. This crop is the only tetraploid $(2 n=4 x=28)$ species of commercial wheat that is widely cultivated in Mediterranean climates with more than $50 \%$ of global wheat production (FAOSTAT 2013). In these regions, yield performance is significantly constrained by drought and heat stress because of unpredictable seasonal events at

*Corresponding author; E-mail: alietminan55@yahoo.com 
the end of growth cycle (Loss and Siddique 1994). Relative yield performance in the both non-stressed and drought-stressed environments can be used as an important indicator of drought tolerance in breeding programs. Based on the theory suggested by Fernandez (1993), genotypes can be categorized into four groups; (1) group A with high performance under both non-stress and stress environments; (2) group B with high yield in non-stress environments; (3) group $\mathrm{C}$ with high yield in stress environments and (4) group D with low yield in both environments. Accordingly, several yield-based drought tolerance criteria, such as stress susceptibility index (SSI), stress tolerance index (STI), yield stability index (YSI), relative drought index (RDI), geometric mean productivity (GMP), mean productivity (MP), tolerance (TOL), harmonic mean (HM), yield index (YI), stress susceptibility percentage index (SSPI), abiotic stress tolerance index (ATI), modified stress tolerance index (MSTI) and integrated selection index have been suggested to characterize the drought tolerance of genotypes (Fischer and Maurer 1978; Rosielle and Hamblin 1981; Bouslama and Schapaugh 1984; Fernandez 1993; Gavuzzi et al. 1997; Farshadfar and Sutka 2002; Moosavi et al. 2008).

The efficiency of suggested indicators depends on the objective of selection and targeted environment. For increasing the yield performance of each genotype, it is necessary to define discriminating criteria with different methods like cluster analysis, factor analysis, principal components analysis, biplot and regression models (Tahmasbi et al. 2013; Khalili et al. 2014; Bahrami et al. 2014; Ravari et al. 2015). Recently, artificial neural network (ANN) analysis suggested as one of the suitable methods, which may prove to be useful in the classifying of drought tolerance indices. The most common ANN model is the multilayer-perceptron (MLP), which has three parts as input, hidden and output layers (Gholipoor et al. 2012). Also, the mean square error (MSE) and the coefficient of determination $\left(R^{2}\right)$ between the predicted and actual values are widely used as other indices for determining the validity of the model (Ravari et al. 2015). Despite ANN was used to predict grain yield in the different crops (Shekoofa et al. 2014; Safa et al. 2015), but this model has not been used for assessment of crop drought tolerance earlier. Hence, the objective of the present study was to determine efficiency of the ANN method to selection of the best drought tolerance criteria.

\section{Materials and Methods}

\section{Plant materials and experimental set up}

In this study, 25 durum wheat genotypes - selected from the breeding program at the Dryland Agricultural Research Institute - were evaluated under two distinct environments (rainfed and supplemental irrigation conditions) during two consecutive years (2011-2012 and 2012-2013). Detailed information about these materials are shown in Table S1*. The trials were conducted at Sararood Research Station in Kermanshah $\left(34^{\circ} 19^{\prime} \mathrm{N}, 47^{\circ} 17^{\prime} \mathrm{E} ; 1351 \mathrm{~m}\right.$ above sea level), which is representative of rainfed environments with moderate winters and annual mean precipitation of $425 \mathrm{~mm}$, mean tempera-

*Further details about the Electronic Supplementary Material (ESM) can be found at the end of the article. 
ture of $11.5^{\circ} \mathrm{C}$ per year. Detailed agro-climate information for each year are in Table S2. For supplemental irrigation two irrigations, each of $25 \mathrm{~mm}$ were applied at flowering and grain filling stages to cope with terminal drought stress, a common feature in western Iran. For each environment, experimental layout was a randomized complete block design with three replications. Plot size was $7.2 \mathrm{~m}^{2}$ (six rows, $6 \mathrm{~m}$ long and $20 \mathrm{~cm}$ row spacing). Fertilizer rate was applied at planting at $50 \mathrm{~kg} \mathrm{~N} \mathrm{ha}^{-1} 1$ and $50 \mathrm{~kg} \mathrm{P}_{2} \mathrm{O}_{5} \mathrm{ha}^{-1}$. At harvest, grain yield data was collected for each plot $\left(\mathrm{g} \mathrm{plot}^{-1}\right)$.

\section{Statistical analysis}

A combined analysis of variance (ANOVA) was performed for grain yield using SAS PROC GLM (SAS Inc, Cary, USA). The thirteen selection indices including TOL, MP, STI, GMP, SSI, YSI, YI, HM, RDI, $\mathrm{K}_{1} \mathrm{STI}, \mathrm{K}_{2} \mathrm{STI}$, ATI and SSPI were calculated based on grain yields under rainfed (Ys) and supplemental irrigation (Yp) environments (Table S3). Regarding the ANN analysis, the multilayer-perceptron (MLP) model was used. To prevent over fitting in the training phase, the dataset was randomly divided into three sets because of high neurons existing in the hidden layer. So, training the neural network, validation set and testing set were based on $70 \%, 15 \%$ and also $15 \%$, of data, respectively (Bagheri et al. 2012). During training, ANN is trying to converge the global minimum or the maximum neural network performance via the shortest path for yielding the size of the network. With this method, back propagation is performed with the training set. The update rule in the method used here seeks to minimize mean square error over the training set. After an epoch of training, the mean square error of network running of the validation set is computed. In this model, evaluated indices and grain yields (Ys, Yp and average of both grain yields (Y)) were considered as input and output data, respectively. Afterwards, validity of the model was determined by MSE and $R^{2}$. Also, a sensitivity analysis was performed for importance evaluating of each forecaster and independent variables during neural network (NN) processes (Ravari et al. 2015). The relative importance parameter for each index is calculated by dividing the value of its importance by the highest importance value. Consequently, the model with the largest $R 2$ and the smallest MSE was considered the best. The accuracy of repeatability and similarity for yields and yield-based indices were evaluated by the ratio of the standard error and the mean value of the correlations estimates from 1000 bootstrap samples similar to coefficient of variation (CV). A selection index is more accurate when its CV is low (Robert 2002). All implementations were performed using neural network toolbox of MATLAB (Neural Network Toolbox, Natick, USA) and SPSS (SPSS, New York, USA) packages.

\section{Results}

\section{Combined analysis of variance (ANOVA)}

The results of combined ANOVA on grain yield showed that all the main and their interaction effects were statistically significant (Table 1). The relative magnitudes of different source of variation indicated that $30,28.64$ and $9.26 \%$ of the total variation in grain yield 
Table 1. Combined analysis of variance (ANOVA) for grain yield ( $\left.\mathrm{g} \mathrm{plot}^{-1}\right)$ in 25 studied durum wheat genotypes

\begin{tabular}{|l|c|c|c|c|}
\hline \multicolumn{1}{|c|}{ Source of variation } & $\mathrm{df}$ & $\mathrm{SS}$ & MS & TSS (\%) \\
\hline Year $(\mathrm{Y})$ & 1 & 3559897.76 & $3559897.76^{* * *}$ & 30.01 \\
\hline Environment (E) & 1 & 3398480.19 & $3398480.19^{* * *}$ & 28.65 \\
\hline $\mathrm{Y} \times \mathrm{E}$ & 1 & 1099370.08 & $1099370.08^{* * *}$ & 9.27 \\
\hline $\mathrm{R} /(\mathrm{Y} \times \mathrm{E})$ & 6 & 18028.86 & 3004.81 & 0.15 \\
\hline Genotype (G) & 24 & 424496.40 & $17687.35^{* * *}$ & 3.58 \\
\hline $\mathrm{Y} \times \mathrm{G}$ & 24 & 723019.44 & $30125.81^{* * *}$ & 6.09 \\
\hline $\mathrm{E} \times \mathrm{G}$ & 24 & 890013.84 & $37083.91^{* * *}$ & 7.50 \\
\hline $\mathrm{Y} \times \mathrm{E} \times \mathrm{G}$ & 24 & 627652.32 & $26152.18^{* * *}$ & 5.29 \\
\hline Error & 192 & 1123132.80 & 5849.65 & 9.47 \\
\hline Coefficient of variance $(\%)$ & & & 19.04 & \\
\hline
\end{tabular}

***Significant at $P<0.0001$. TSS - percentage relative to total sum of square.

was justified by differences among years, environments and their interaction, respectively. Interactions between years and genotypes explained $6.10 \%$, whereas genotype $\times$ environment interaction accounted for $7.50 \%$ of the total variation. Furthermore, year $\times$ environment $\times$ genotype effect accounted for $5.29 \%$ of the total variation about grain yield. Also, the moderate coefficient of variance was observed for grain yield (19\%).

\section{Response of genotypes to drought stress}

Mean yields of the 25 durum wheat genotypes under irrigated and rainfed environments along with their relative drought effect (RDF) are shown in Tables S3-5. The results showed significant differences among genotypes in each environment and a decreasing trend was occurred due to drought stress. The grain yield declined by 27.12, 49.29 and $41.90 \%$ in the first, second and average two years, respectively. Under irrigated environment, in the first year, the average yield across all the genotypes ranged from $205.97 \mathrm{~g}$ plot $^{-1}$ (corresponding to G24) to 516.68 g plot $^{-1}$ (corresponding to G7), with an average of $262.10 \mathrm{~g} \mathrm{plot}^{-1}$, while under drought environment, it varied between $178.07 \mathrm{~g} \mathrm{plot}^{-1}$ (corresponding to G24) and $315.47 \mathrm{~g} \mathrm{plot}^{-1}$ (corresponding to G8), with an average of 217.27 $\mathrm{g} \mathrm{plot}^{-1}$. The lowest reduction in grain yield was recorded for genotype G20 followed by G22, G3, G12 and G24. However, grain yield increased by 25.86, 6.92 and $2.82 \%$ in G2, G17 and G8, respectively (Table S4). In the second year, response of genotypes to drought stress was different and the grain yield under irrigated environments ranged from $500.65 \mathrm{~g}$ plot $^{-1}$ (corresponding to G25) to $838.80 \mathrm{~g} \mathrm{plot}^{-1}$ (corresponding to G17), with an average of $677.47 \mathrm{~g} \mathrm{plot}^{-1}$. In the second year, grain yield ranged from $145.97 \mathrm{~g} \mathrm{plot}^{-1}$ to $612.50 \mathrm{~g}$ plot $^{-1}$ (G7 and G22, respectively) under rainfed environment. The order of least grain yield reduction was as $\mathrm{G} 14<\mathrm{G} 25<\mathrm{G} 20<\mathrm{G} 22<\mathrm{G} 16<$ other genotypes (Table S5). Across two years the mean grain yield varied between $295.14 \mathrm{~g} \mathrm{plot}^{-1}$ and $508.01 \mathrm{~g} \mathrm{plot}^{-1}$ for irrigated 
and rainfed environments, respectively. Also, the last genotypes except G16 indicated the lowest reduction than other genotypes. The highest grain yield under irrigated/rainfed environments were recorded by genotypes G11/G22, while the lowest values were recorded for G25 and G24, respectively. Furthermore, the lowest RDE was observed for genotype G20 followed by G25, G22, G14 and G2 (Table S6). The ranks of drought tolerance indices for 25 durum genotypes are shown in Tables S7-9. Also, due to the difficulty in identifying drought tolerant genotypes based on a single criteria, the average of sum ranks (ASR) of all indices was calculated to select the superior tolerant genotypes with low ASR values. Based on these results, genotype G11, G8, G15, G7 and G9 in the first year; G22, G23, G20, G8 and G2 in the second year; and G23, G24, G9, G21 and G12 in the average two years were identified as the best tolerant genotypes.

\section{Similarity patterns among drought tolerance indices and their accuracy}

Principal component analysis (PCA)-based biplot was applied to discover the interrelationships among drought tolerance indices. These relationships were assessed by acute and obtuse angles among indices vectors on the biplot. For instance, an acute angle shows a strong positive association and an obtuse angle shows a negative correlation; furthermore a 180 angle would result if there was negative correlation, whereas a 90 angle indicates if there was no correlation among indices. As shown in Fig. S1, most of correlations among indices were significantly positive. Moreover, the correlations among MP, GMP, STI and two modified STI $\left(\mathrm{K}_{1} \mathrm{STI}\right.$ and $\left.\mathrm{K}_{2} \mathrm{STI}\right)$ indices were positively significant (Fig. S1A-C), suggesting a high similarity among these tolerance indices for genotypes ranking. Also, Ys strongly correlated with YSI index. Four tolerance indices, TOL, SSI, SSPI and ATI, had the significant positive correlation with each other. In addition, a repeatable relationship among aforesaid indices with grain yield under irrigated (Yp) environment expressed that increased Yp is related to drought susceptibility. Additionally, accuracy and repeatability of measured grain yields and indices were investigated using CV values resulted from repeated bootstrap samples which are presented in Table 2 . In the first year, the lowest CV values were estimated for Ys, Y, MP, GMP, HM and YI. In the second year, Y, MP, Yp, GMP and HM indicated the lowest CV values. Regarding the averages of two years, Y, MP, GMP and HM were selected as the most accurate indices.

\section{Identification of the best indices using artificial neural network (ANN) analysis}

The ANN analysis was performed for each year and also average two years. In all of the ANN analyzes, the input and output layers had 13 neurons (representing drought indices) and one layers (corresponding to the yield performance of genotypes), respectively. The number of neurons in the hidden layer was defined using a trial-and-error approach that was started with one and then consecutively increased. Then, the number of learning cycles (epoch), learning rate and momentum were constants 10,000, 0.8 and 0.6, respectively. The results of ANN showed different networks across two years (Table S10). In the first year, a network with eight hidden layers selected in the $10^{\text {th }}$ training step and estab- 
Table 2. Coefficients of variation estimated over 1000 bootstrap runs for yield-based drought tolerance indices between different growing seasons

\begin{tabular}{|l|c|c|c|}
\hline \multicolumn{1}{|c|}{ Index } & First year & Second year & Average of two years \\
\hline $\mathrm{Y}_{\mathrm{P}}$ & 4.14 & 2.07 & 2.01 \\
\hline $\mathrm{Y}_{\mathrm{S}}$ & 2.53 & 1.87 & 1.37 \\
\hline $\mathrm{Y}$ & 1.51 & 4.77 & 2.70 \\
\hline STI & 4.48 & 4.96 & 2.96 \\
\hline SSI & 44.17 & 6.46 & 5.99 \\
\hline MP & 2.53 & 1.87 & 1.37 \\
\hline GMP & 2.37 & 2.49 & 1.46 \\
\hline TOL & 15.50 & 7.12 & 6.88 \\
\hline HM & 2.27 & 3.25 & 1.69 \\
\hline YI & 6.62 & 5.64 & 2.70 \\
\hline YSI & 1.51 & 4.77 & 3.90 \\
\hline K $_{1}$ STI & 11.43 & 7.01 & 5.97 \\
\hline K $_{2}$ STI & 6.52 & 13.59 & 8.19 \\
\hline SPPI & 15.49 & 7.12 & 6.87 \\
\hline ATI & 14.51 & 6.57 & 7.03 \\
\hline RDI & 6.62 & 5.64 & 3.89 \\
\hline
\end{tabular}

$\mathrm{Y}_{\mathrm{P}}$, grain yield in supplemental irrigated environment; $\mathrm{Y}_{\mathrm{S}}$, grain yield in rainfed environment; $\mathrm{Y}$, average of both grain yields; STI, stress tolerance index; SSI, stress susceptibility index; MP, mean product; GMP, geometric men production; YI, yield index; YSI, yield stability index; TOL, tolerance index; HM, harmonic mean; $\mathrm{K}_{1} \mathrm{STI}$ and $\mathrm{K}_{2} \mathrm{STI}$, modified stress tolerance index; SSPI, stress susceptibility percentage index; ATI, abiotic stress tolerance index and RDI, relative drought index.
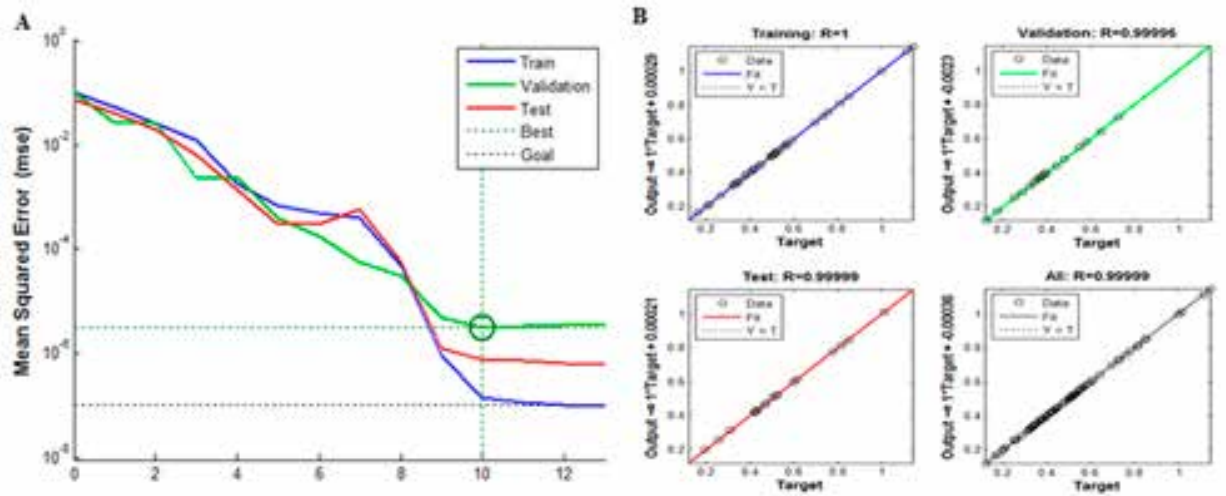

Figure 1. Change in the MSE during the training steps (A) and predicted values of tolerance by a multilayer perceptron neural network versus observed values intesting, training and validation phases (B) based on the first year dataset (2011-2012) 
lished minimum error, whereas in the second year the network established with eight hidden layers in the $7^{\text {th }}$ training step. In average two years, minimum error occurred when the network was selected with eight hidden layers in $6^{\text {th }}$ training step (Figs $1-3$ ). In all of analyzes, the values of $R 2$ in the training and testing phases indicated that the neural network had been trained and revealed good performance in predicting the responses of genotypes to drought stress based on measured indices. As shown in Table S7, when Yp and Ys selected as the dependent variables, the effective indices in each environment were different to each other. Hence, the average of grain yield in the both irrigated and rainfed environments, was considered as dependent variable. Accordingly based on the relative importance parameter, SSI, HM, ATI, YSI and GMP in the first year; HM, YSI, MP, SSI and RDI in the second year; TOL, YI, YSI, SSI and HM in the average two years were selected as the best indices (Table 3).
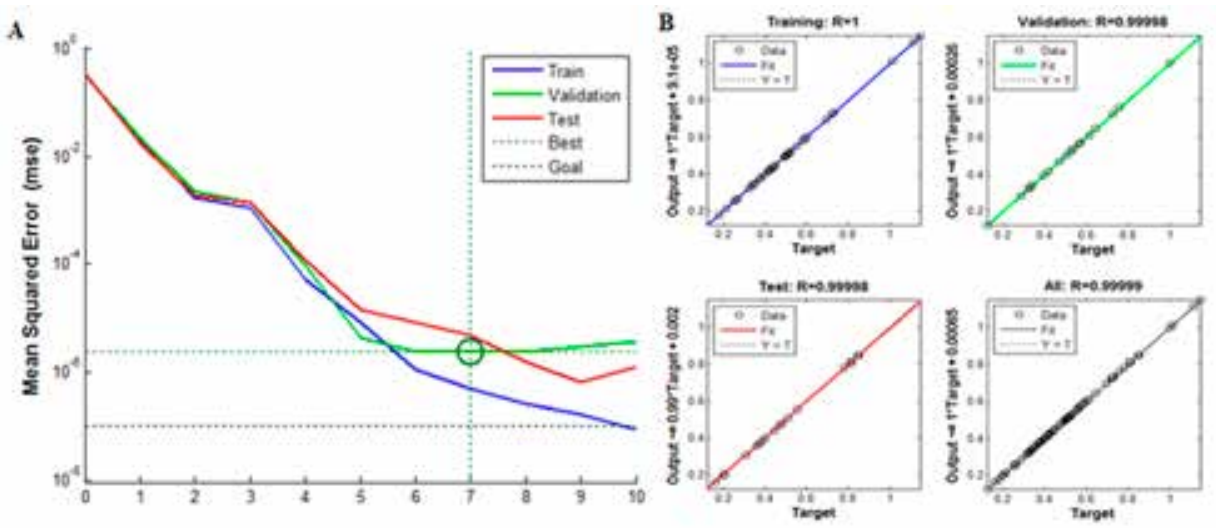

Figure 2. Change in the MSE during the training steps (A) and predicted values of tolerance by a multilayer perceptron neural network versus observed values intesting, training and validation phases (B) based on the second year dataset (2012-2013)
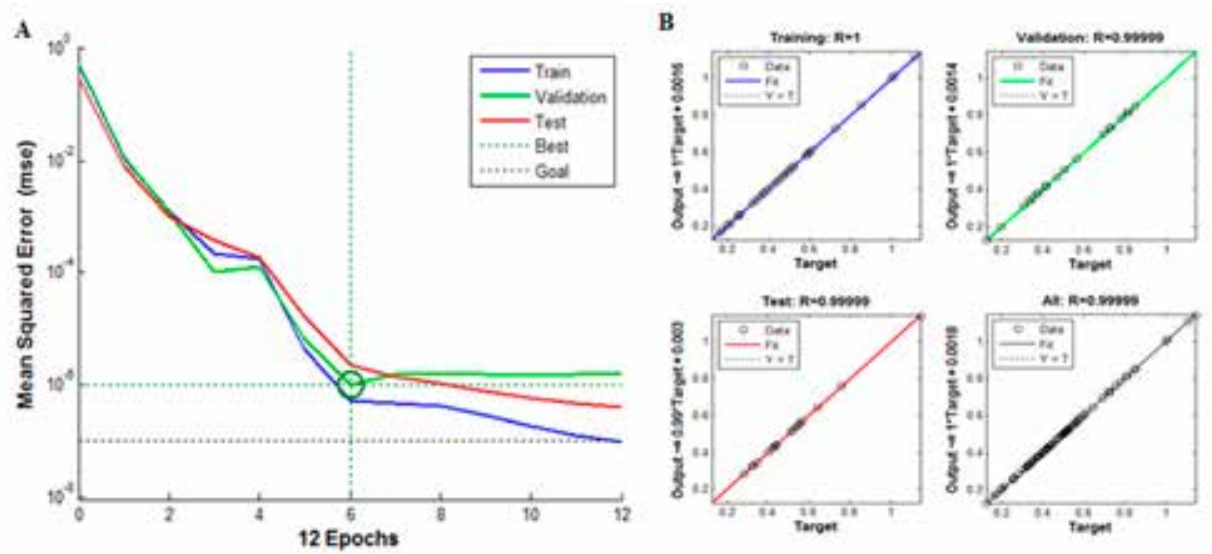

Figure 3. Change in the MSE during the training steps (A) and predicted values of tolerance by a multilayer perceptron neural network versus observed values intesting, training and validation phases (B) based on average two years 


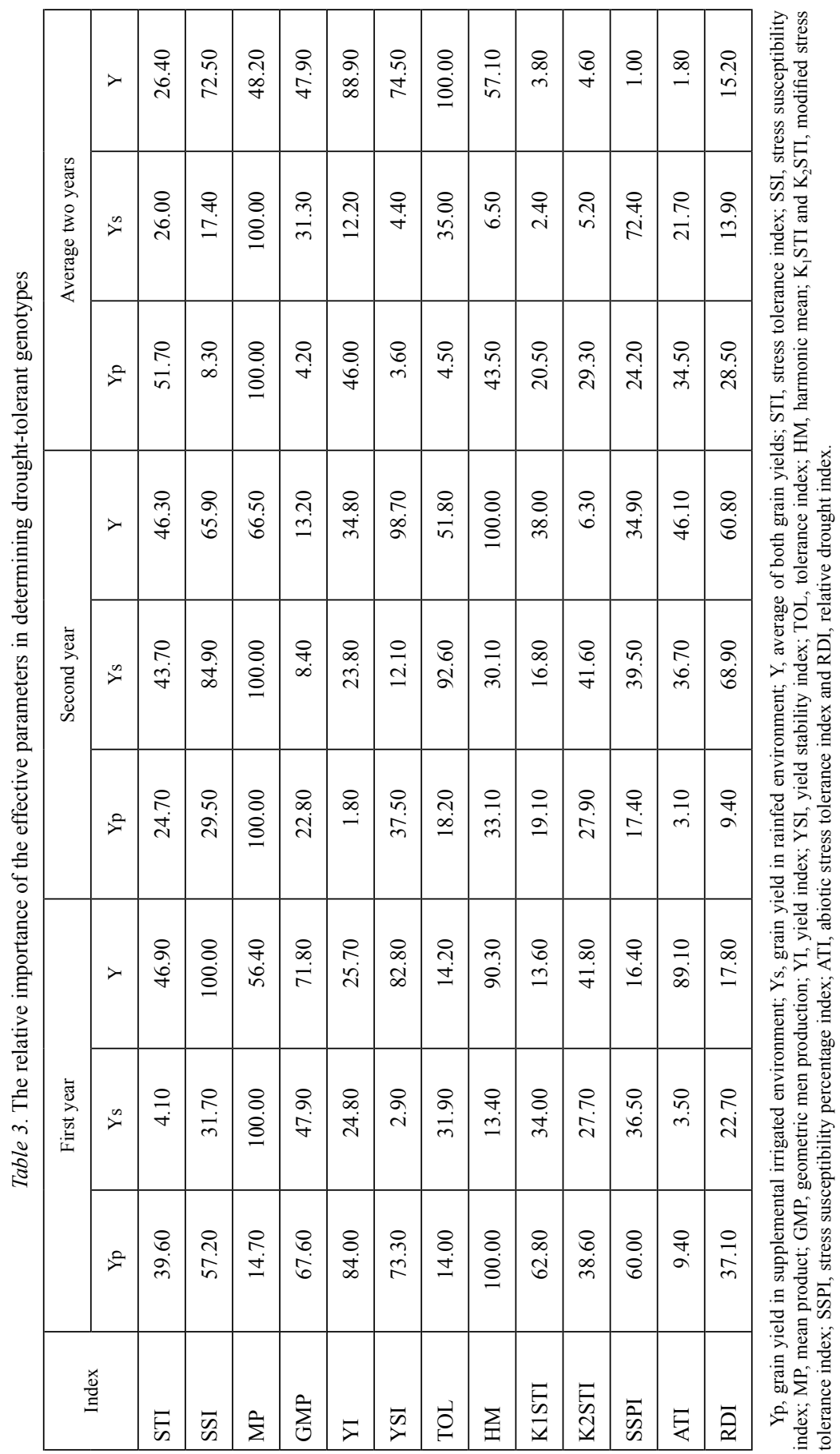




\section{Discussion}

In this work, we evaluated the yield performance and tolerance of 25 durum wheat genotypes under two different environments (irrigated and rainfed environments). Our results indicated the wide variability in the studied genotypes in response to drought stress (Table 1). Also, a large variation explained by environments $(28.65 \%$ of the total sum of square) revealed that environments were diverse with large differences among environmental means causing most of the variation in grain yield. Grain yield is a complex quantitative trait, which is controlled by multiple genes and is highly influenced by various environmental factors (Shi et al. 2014). Hence, the detected different responses to drought stress in this study may be resulted from genetic variability among genotypes or environmental conditions and even presence of the interaction among genotypes and environments. To identify the best drought tolerant genotypes, we used several yield-based drought tolerance and susceptibility indices. Regarding obtained results, selection based on each index was different, hence, we used the ASR approach to classify the best genotypes. Our results showed G23, G24, G9, G21 and G12 in the both studied years as the best tolerant genotypes. Likewise, Khalili et al. (2012), Naghavi et al. (2013) and PourSiahbidi and Pour-Aboughadareh (2013), in the different crops, used the ASR method to classify the best drought-tolerant genotypes based on their yield performance and tolerance indices ranking under stress and non-stress conditions. The accuracy of different drought tolerance indices is an important issue that should be measured during estimating of similarity for tolerance indices in drought-prone environments. This parameter can be helpful for recognizing the most efficient and accurate indices across different years or different drought stress levels (Mohammadi 2016). Among different indices, MP, GMP and $\mathrm{HM}$ with the lowest $\mathrm{CV}$ values were identified as the most accurate indices (Table 2). Furthermore, the principal component analysis (PCA) as the relevant multivariate analysis used to display relationships among different indices. According to PCA-based biplot, consistent pattern of correlations was observed among TOL, SSI, SSPI and ATI, indicating these indices can be used interchangeably across different environments.

Recently, artificial neural network (ANN) approach as a novel analytical method has been served to predict the different biological aspects. Several advantages of ANN make it valuable and attractive for a forecasting task: (i) as opposed to the traditional model, ANN is data-driven self-adaptive technique that this somewhat provides priori assumptions about the models for probable problems during the study, (ii) ANN can generalize after learning the data presented to them (a sample) and also it can correctly infer the unseen part of a population even if the sample data contain noisy information and (iii) ANN is universal functional approximate. It has been shown that a network can approximate any continuous function to any desired accuracy, and this technique can also solve linear and nonlinear functions (Khashei and Bijari 2010). Based on our results, the values of $R 2$ in the all analyzes indicated that the neural network had been trained and revealed good performance in predicting the responses of genotypes to drought stress based on measured indices. Furthermore, the relative important factor indicated that repeatability of the indices affected by environmental conditions. However, several indices like TOL, 
YI, YSI, SSI and HM were identified as the best indices to recognizing the drought-tolerant genotypes (Table 3). Previously, Ravari et al. (2015) used ANN to predict grain yield in wheat and also to assess salinity indices for identifying tolerant genotypes in saline lands in Iran. They used several indices as input and grain yield as the output variable in the model, and then confirmed the accuracy of the ANN model. Moreover, the results of their study revealed that yield stability index (YSI), geometric mean production (GMP), mean product (MP) and stress tolerance index (STI) were selected as the best indices to predict drought-tolerant varieties.

The ANN analysis would be expected for other researches to establish an intelligent agricultural information system to help experts and also to improve agricultural technologies (Shekoofa et al. 2014). In recent year, agricultural and biological studies have used various techniques of data mining for analyzing large data collections and establishing useful classification patterns within the data sets. However, data mining methods are still expected to bring more efficient results (Hsiao et al. 2006). Intelligent data mining and knowledge discovery using ANN and feature selection algorithms have become the important revolutionary issues in prediction and modeling (Ebrahimi et al. 2010). In a different study, Shekoofa et al. (2014) used an ANN method to optimize the physiological and agronomic characters for estimation of grain yield in maize. They used the different characters such as, season duration, days to silking, sowing date-location, soil type, fertilizers, soil $\mathrm{pH}$, hybrid type, grain filling period, plant density, plant height, defoliation, leaf dry weight, stem dry weight, kernel growth rate, kernel number and weight, grain yield, kernel water content, and cob dry weight as input variables and grain yield as the output variable in the model. Finally, their results confirmed the accuracy of the ANN method and several characters like sowing date-location, the number of kernels, kernels weight and season duration were introduced as the most important characters that had the highest effect on yield performance.

\section{Conclusion}

The ANN approach provides a practical solution for predicting drought tolerant genotypes in a fast, inexpensive, accurate and objective way. The combination of artificial intelligence and agricultural practices will continue to be an interesting area of research in future agronomy and plant breeding programs. In conclusion, this study provides evidences that ANN would be able to plays an important role in selection of drought-tolerant genotypes and it could be valuable in various other biological contexts.

\section{References}

Bagheri, S., Gheysari, M., Ayoubi, S., Lavaee, N. 2012. Silage maize yield prediction using artificial neural networks. Int. J. Plant Prod. 19:77-96.

Bahrami, F., Arzani, A., Karimi, V. 2014. Evaluation of yield-based drought tolerance indices for screening safflower genotypes. Agron. J. 106:1219-1224.

Bouslama, M., Schapaugh, W.T. 1984. Stress tolerance in soybean: 1. Evaluation of three screening techniques for heat and drought tolerance. Crop Sci. 24:933-937. 
Ebrahimi, M., Ebrahimie, E. 2010. Sequence-based prediction of enzyme thermostability through bioinformatics algorithms. Curr. Bioinform. 5:195-203.

Farshadfar, E., Sutka, J. 2002. Multivariate analysis of drought tolerance in wheat substitution lines. Cereal. Res. Commun. 31:33-39.

Fernandez, G.C.J. 1993. Effective selection criteria for assessing plant stress tolerance. In: Kuo, C.G. (ed), Proceeding of the AFCTWS Adaptation of food crops to temperature and water stress; Shanhua, Taiwan, pp. 257-270.

Fischer, R.A., Maurer, R. 1978. Drought resistance in spring wheat cultivars: 1. Grain yield response. Aust. J. Agric. Res. 29:897-912.

Food and Agriculture Organization, FAO. 2013. http://www.fao.org/faostat/en/

Gavuzzi, P., Rizza, F., Palumbo, M., Campaline, R.G., Ricciardi, F.L., Borghi, G. 1997. Evaluation of field and laboratory predictors of drought and heat tolerance in winter cereals. Plant Sci. 77:523-531.

Gholipoor, M., Rohani, A., Torani, S. 2012. Optimization of traits to increasing barley grain yield using an artificial neural network. Int. J. Plant. Prod. 7:1-18.

Hsiao, H.W., Tasi, M.S., Wang, S.C. 2006. Spatial data mining of colocation patterns for decision support in agriculture. Asian Journal of Health and Info. Sci. 1:61-72.

Khalili, M., Naghavi, M.R., Pour-Aboughadareh, A.R., Talebzadeh, S.J. 2012. Evaluating of drought stress tolerance based on selection indices in spring canola cultivars (Brassica napus L.). J. Agric. Sci. 4:78-85.

Khalili, M., Pour-Aboughadareh, A., Naghavi, M.R., Mohammad-Amini, E. 2014. Evaluation of drought tolerance in safflower genotypes based on drought tolerance indices. Not. Bot. Horti. Agrobot. Cluj-Napoca. 42:214-218.

Khashei, M., Bijari, M. 2010. An artificial neural network (p, d, q) model for time series forecasting. Expert. Syst. Appl. 37:479-489.

Loss, S.P., Siddique, K.H.M. 1994. Morphological and physiological traits associated with wheat yield increases in Mediterranean environments. Adv. Agron. 52:229-276.

Mohammadi, R. 2016. Efficiency of yield-based drought tolerance indices to identify tolerant genotypes in durum wheat. Euphytica 211:71-89.

Moosavi, S.S., Yazdi-Samadi, B., Naghavi, M.R., Zali, A.A., Dashti, H., Pourshahbazi, A. 2008. Introduction of new indices to identify relative drought tolerance and resistance in wheat genotypes. Desert. 12:165-178.

Naghavi, M.R., Pour-Aboughadareh, A.R., Khalili, M. 2013. Evaluation of drought tolerance indices for screening some of corn (Zea mays L.) cultivars under environmental conditions. Not. Sci. Biol. 5:388-393.

Pour-Siahbidi, M.M., Pour-Aboughadareh, A. 2013. Evaluation of grain yield and repeatability of drought tolerance indices for screening chickpea (Ciceraritinum L.) genotypes under rainfed conditions. Iranian J. Genetics. Plant. Breed. 2:28-37.

Ravari, S.Z., Dehghani, H., Naghavi, H. 2015. Assessment of salinity indices to identify Iranian wheat varieties using an artificial neural network. Ann. Appl. Biol. 168:185-194.

Robert, N. 2002. Comparison of stability statistics for yield and quality traits in bread wheat. Euphytica. 128:333-341.

Rosielle, A.A., Hamblin, J. 1981. Theoretical aspects of selection for yield in stress and non-stress environment. Crop. Sci. 21:943-946.

Safa, M., Samarasinghe, S., Nejat, M. 2015. Prediction of wheat production using artificial neural networks and investigating factors affecting it: case study in Canterbury province, New Zealand. J. Agr. Sci. Tech. 17:791-803.

Shekoofa, A., Emam, Y., Shekoufa, N., Ebrahimi, M., Ebrahimie, E. 2014. Determining the most important physiological and agronomic traits contributing to maize grain yield through machine learning algorithms: A new avenue in intelligent agriculture. Plos One. 9:e97288.

Shi, C., Dong, B., Qiao, Y., Guan, X., Si, F., Zheng, X., Liu, M. 2014. Physiological and morphological basis of improved water-use-efficiency in wheat from partial root-zone drying. Crop Sci. 54:2745-2751.

Tahmasbi, G., Heydarnezhadian, J., Pour-Aboughadareh, A. 2013. Evaluation of yield and yield components in some of promising wheat lines. Intl. J. Agri. Crop Sci. 20:2379-2384. 


\section{Electronic Supplementary Material (ESM)}

Electronic Supplementary Material (ESM) associated with this article can be found at the website of CRC at https://akademiai.com/loi/0806

Electronic Supplementary Table S1. Names/pedigree for 25 tested genotypes

Electronic Supplementary Table S2. Description of the durum wheat testing years under rainfed conditions

Electronic Supplementary Table S3. Drought tolerance/sensitive indices measured in this study

Electronic Supplementary Table S4. Drought tolerance indices for 25 durum wheat genotypes in the first year (2011-2012)

Electronic Supplementary Table S5. Drought tolerance indices for 25 durum wheat genotypes in the second year (2012-2013)

Electronic Supplementary Table S6. Drought tolerance indices for 25 durum wheat genotypes based on average of two years

Electronic Supplementary Tables S7. Rank of drought tolerance indices for 25 durum wheat genotypes in the first year (2011-2012)

Electronic Supplementary Table S8. Rank of drought tolerance indices for 25 durum wheat genotypes in the second year (2012-2013)

Electronic Supplementary Table S9. Rank of drought tolerance indices for 25 durum wheat genotypes based on average of two years

Electronic Supplementary Table S10. Error variations with different number of neurons in the training, testing and validating phases

Electronic Supplementary Figure S1. Biplot drawn based on the two first components obtained from PCA using the drought tolerance/sensitive indices and grain yield of 25 durum wheat genotypes under supplemental irrigation (Yp) and rainfed (Ys) environments in the first year (A), second year (B), and average two years (C) 